\title{
Homogeneous Pd-catalyzed Heck coupling in $\gamma$-valerolactone as a green reaction medium: a catalytic, kinetic, and computational study
}

Dániel Fodor, ${ }^{\mathrm{a}}$ Tamás Kégl, ${ }^{\mathrm{b}}$ József M. Tukacs, ${ }^{\mathrm{a}}$ Attila K. Horváth ${ }^{\mathrm{b}, *}$ and László T. Mikaa,

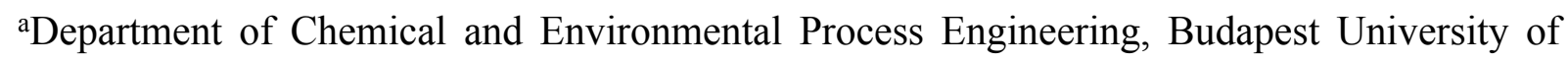
Technology and Economics, Müegyetem rkp. 3., H-1111Budapest, Hungary.

${ }^{b}$ Department of Inorganic Chemistry and MTA-PTE Research Group for Selective Chemical Syntheses, University of Pécs, Ifjúság u. 6., H-7624 Pécs, Hungary.

*e-mail László T. Mika: laszlo.t.mika@mail.bme.hu

*e-mail Attila K. Horváth: horvatha@gamma.ttk.pte.hu

Electronic Supplementary Information (SI)

Number of Pages: 25

Number of Figures: 3

Number of Tables: 1

\section{Source of Chemicals}

Gamma-valerolactone, iodobenzene, 4-iodotoluene, 4-iodoanizole, 4-chloro iodobenzene, 4chloro iodobenzene, 4-fluoro iodobenzene, 4-trifluoromethoxy, 4-nitro iodobenzene, styrene, 4methoxystryrene, 4-chlorostrytene, 4-acetoxystyrene, p-xylene, stylbene, and 1,1-diphenyl ethylene were purchased from Sigma-Aldrich Kft., Budapest, Hungary. 

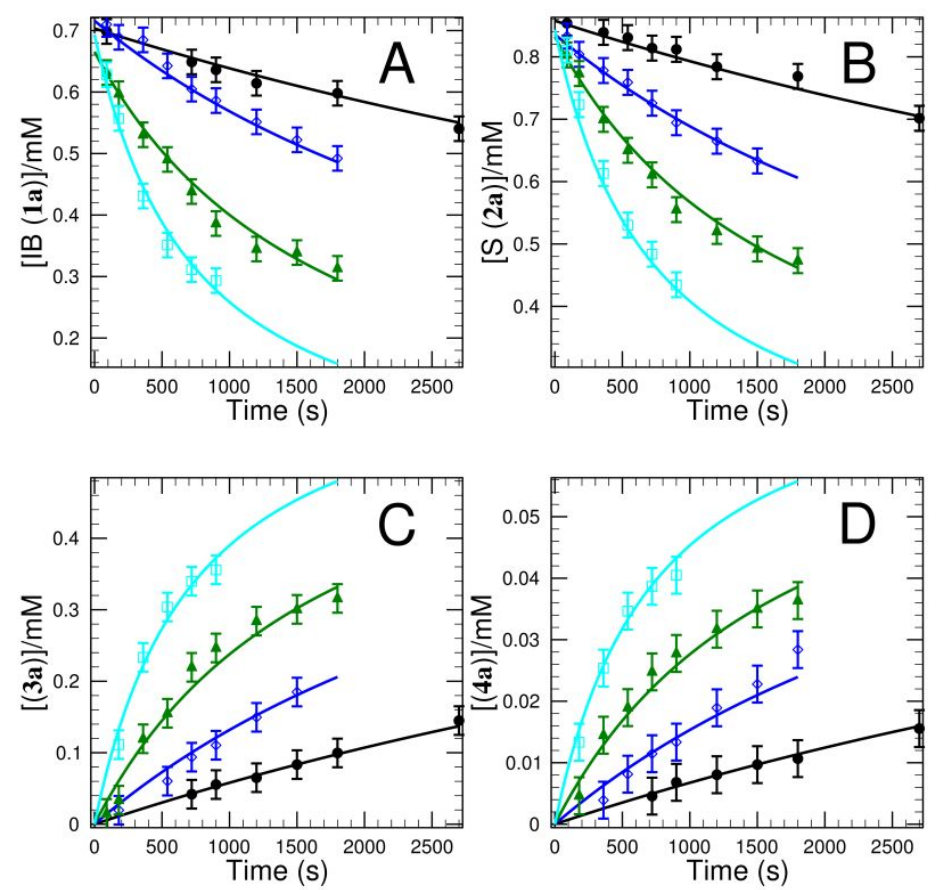

Figure S1. Experimental and fitted kinetic curves obtain in the iodobenzene-styrene reaction at different temperature using DMF as solvent. Initial conditions are as follows: $[1 \mathrm{a}]_{0}=0.7 \mathrm{mM}$, $[2 \mathrm{a}]=0.84 \mathrm{mM},[\mathrm{C}]_{0}=0.78 \mu \mathrm{M}$. T $/{ }^{\circ} \mathrm{C}=90$ (black), 100 (blue), 110 (green), 120 (cyan).

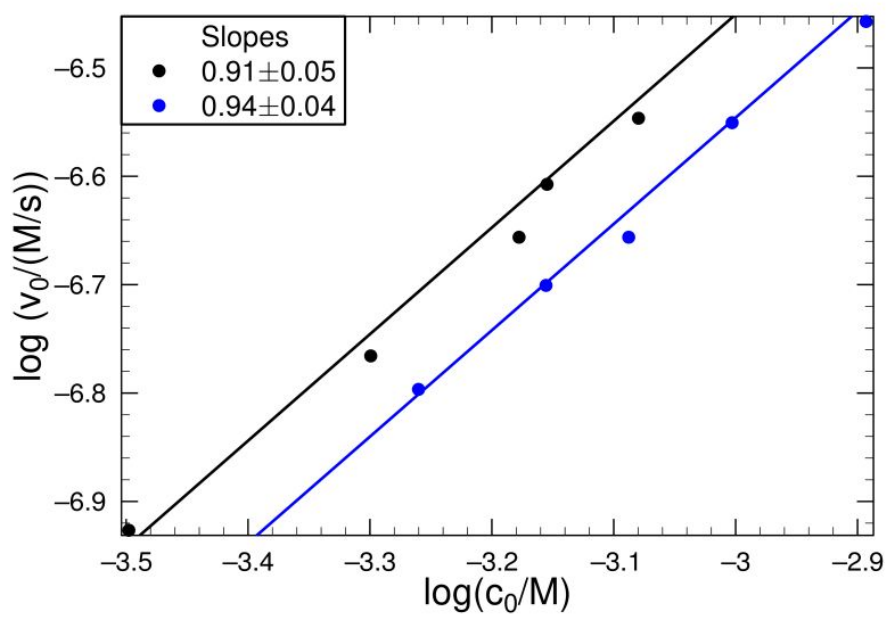

Figure S2. Initial rate studies determining the formal kinetic orders of iodobenzene (black color) and styrene (blue color), where $v_{0}$ values correspond to $d[\mathrm{IB}] / d t$ at $\mathrm{t}=0 \mathrm{~s}$ time point in case of the experimental curves and $c_{0}$ refers to the initial concentrations of 1a (black circles) and 2a (blue circles). The straight lines are obtained for linear regression. Initial conditions kept constant at 
these series of runs are as follows: (black circles) $[\mathbf{2 a}]_{0}=0.82 \mathrm{mM}$, [cat $]_{0}=0.68 \mu \mathrm{M}$ and $\mathrm{T}=105$ ${ }^{\circ} \mathrm{C}$; (blue circles) $[\mathbf{1 a}]_{0}=0.67 \mathrm{mM}$, [cat $]_{0}=0.68 \mu \mathrm{M}$ and $\mathrm{T}=378 \mathrm{~K}$.

\section{Calculations}

$$
\text { Conversion of } \mathbf{1 a}=\frac{\text { mol of converted iodobenzene }(\mathbf{1 a})}{\text { total mol of iodobenzene }(\mathbf{1 a})}
$$

$$
\text { Yield of 3a }=\frac{\text { mol of trans }- \text { stilbene }(\mathbf{3 a})}{\text { total mol of iodobenzene (1a) }}
$$

$$
\text { Yield of } \mathbf{4 a}=\frac{\text { mol of } 1,1-\text { diphenylethylene }(\mathbf{4 a})}{\text { total mol of iodobenzene }(\mathbf{1 a})}
$$

Selectivity of $\mathbf{3 a}=\frac{\text { mol of trans }- \text { stilbene }(\mathbf{3 a})}{\text { mol of trans }- \text { stilbene }(\mathbf{3 a})+\text { mol of } 1,1-\text { diphenylethylene (4a) }}$

$$
\text { Selectivity of } \mathbf{4 a}=\frac{\text { mol of } 1,1-\text { diphenylethylene }(\mathbf{4 a})}{\text { mol of trans }- \text { stilbene }(3 \mathbf{a})+\text { mol of } 1,1-\text { diphenylethylene }(\mathbf{4 a})}
$$

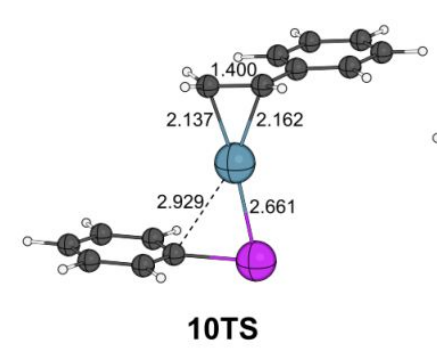

10TS

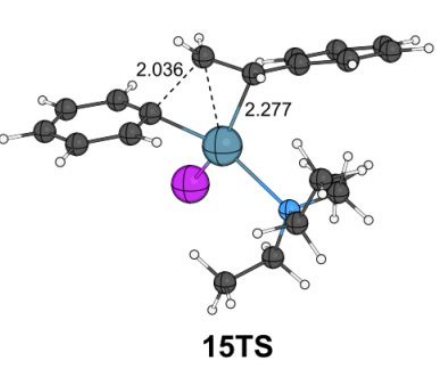

$15 \mathrm{TS}$
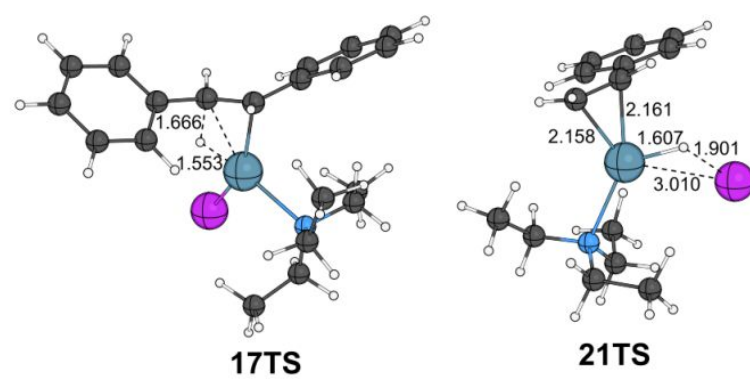

21TS

Figure S3. Oxidative addition (10TS), carbon-carbon coupling (15TS), $\beta$-elimination (17TS), and HI elimination (21TS) transition states of the proposed pathway. Bond length are given in $\AA$.

Table S1. Cartesian coordinates, absolute Gibbs free energies, and number of imaginary frequencies $\left(\mathrm{N}_{\mathrm{imag}}\right)$ of optimized structures occurring in this study.

\section{stilbene}

C $\quad 0.34123512275126 \quad-3.73666985000829 \quad-1.83629976697854$ 

C 0.34211533743153
C 1.14765461494840
C 1.10809929914165
H $\quad-0.27225621121782$
C 1.91233538620273
H 1.17901640117398
C 1.89814488205108
H 1.08840850131609
H 2.52669733371302
H 2.49817298952927
C -2.98651497833496
C -2.21643466007739
C -3.02845287737242
H -2.19796569397804
H -3.63186855276100
C -1.46209568092138
C -2.28755670435597
H -2.31023625661284
C -1.51600456910220
H -0.94052659372184
H $\quad-3.55948990035324$
C -0.63675896577250
H -0.10245922848735
C -0.48481346580997
H -1.02658496138006
$\mathrm{G}=-339052.2297 \mathrm{kcal} / \mathrm{mol}$
$\mathrm{N}_{\text {imag }}=0$

\section{PhI}

C -0.19955272062990

C -1.35155416404790

C 0.94822278755926

H $\quad-2.24797528324861$

I 2.70689522693916

C -1.35443225156636

C 0.96286592900526

$\mathrm{H} \quad-2.25394850499175$

H 1.86180608560218

$-2.96132438702665$

$-3.31470508008274$

$-2.60927510622601$

$-3.58989808340848$

$-2.06977141516985$

$-3.31562571979011$

$-2.60478559567982$

$-3.59175006146420$

$-2.32876006960158$

$-2.96079432194698$

C -0.19778323345666

$-2.95854053498124$

H -0.19080481169144

$-2.96077004362233$

H -0.19849908547327

$\mathrm{G}=-332120.9834 \mathrm{kcal} / \mathrm{mol}$

$\mathrm{N}_{\text {imag }}=0$

\section{HI}

$\begin{array}{lll}\mathrm{H} & 0.00000000000000 & 0.00000000000000\end{array}$

I $0.00000000000000 \quad 0.00000000000000$

$\mathrm{G}=-187241.9243 \mathrm{kcal} / \mathrm{mol}$

$\mathrm{N}_{\text {imag }}=0$

styrene
$-1.51465908279577$

$-2.91399205059314$

$-2.23420112665201$

$-0.68574404424117$

$-3.63216354089561$

$-3.19090815154617$

$-3.29844587418174$

$-1.96365116621242$

$-4.45885025098435$

$-3.86239467031330$

1.39793714658796

0.68510100293851

1.01826558369531

1.00210381024375

1.57894319731160

$-0.43436101482880$

$-0.08539095623675$

$-0.39074174860530$

$-0.79952880288057$

$-1.65965357335471$

2.25790835978681

$-1.22506963129408$

$-2.04386889386702$

$-1.04528916985934$

$-0.23265408424315$
$-3.43344511129605$

$-2.72740683140647$

$-2.72261714625510$

$-3.27724692805474$

$-3.78494071600727$

$-1.33213722035750$

$-1.32755849907819$

$-0.78900513215336$

$-0.78602093077964$

$-0.63708500215093$

0.44992897313346

$-4.51860983659422$ 


\begin{tabular}{|c|c|c|c|}
\hline C & -0.48762339150282 & 0.00190838992904 & -4.24967775042292 \\
\hline $\mathrm{C}$ & -1.64986566464408 & -0.35454707567228 & -3.55833649554497 \\
\hline $\mathrm{C}$ & 0.65609895982572 & 0.35275787052162 & -3.53333269873885 \\
\hline $\mathrm{H}$ & -2.54562461382184 & -0.62934990734767 & -4.10985285908649 \\
\hline $\mathrm{H}$ & 1.56543392542592 & 0.63168620295289 & -4.05969900089786 \\
\hline $\mathrm{C}$ & -1.66852520882861 & -0.36021618558758 & -2.16826165511872 \\
\hline $\mathrm{C}$ & 0.63546297178790 & 0.34649567446416 & -2.13985416322755 \\
\hline $\mathrm{H}$ & -2.58129613239989 & -0.64016375940790 & -1.65012563734178 \\
\hline $\mathrm{H}$ & 1.53055938404800 & 0.62112156044208 & -1.58552769019484 \\
\hline $\mathrm{C}$ & -0.52386748625992 & -0.00908781018814 & -1.42852164790816 \\
\hline $\mathrm{H}$ & -0.47681714773726 & 0.00517079211176 & -5.33641805471911 \\
\hline $\mathrm{C}$ & -0.48802383944881 & 0.00194891248444 & 0.03825009934163 \\
\hline $\mathrm{H}$ & 0.47220328174191 & 0.29684138523656 & 0.46401301180248 \\
\hline $\mathrm{C}$ & -1.48268725215337 & -0.30349201101907 & 0.88280783730475 \\
\hline $\mathrm{H}$ & -2.46974552899962 & -0.60662422818982 & 0.54301734946506 \\
\hline $\mathrm{H}$ & -1.33372486903323 & -0.25771696773006 & 1.95753125728735 \\
\hline \multicolumn{4}{|c|}{$\mathrm{G}=-194164.5811 \mathrm{kcal} / \mathrm{mol}$} \\
\hline \multicolumn{4}{|c|}{$\mathrm{N}_{\mathrm{imag}}=0$} \\
\hline \multicolumn{4}{|c|}{$\mathrm{NEt}_{3}$} \\
\hline $\mathrm{N}$ & -0.089148480 & -0.25290066645900 & 0.32736843 \\
\hline $\mathrm{H}$ & 1.02729238581437 & 1.50009247816054 & 0.44750553683473 \\
\hline $\mathrm{H}$ & -1.02793777783172 & -2.85320297649900 & -0.15901476020352 \\
\hline $\mathrm{H}$ & -1.61714982015523 & -0.56009566412463 & -1.12744076508376 \\
\hline $\mathrm{H}$ & -2.72935355172151 & -2.43809204478549 & 0.07746211281424 \\
\hline $\mathrm{C}$ & -1.70467024909012 & -2.15673565099903 & 0.34726038134600 \\
\hline $\mathrm{H}$ & -0.51267515633092 & 2.77183586877226 & 1.90206082759928 \\
\hline $\mathrm{C}$ & -0.02537308866520 & 1.20326022210581 & 0.48890375145231 \\
\hline $\mathrm{C}$ & -1.43415667591077 & -0.70506681293701 & -0.04274168593828 \\
\hline $\mathrm{H}$ & -0.08066967951812 & 1.21565230196607 & 2.65264124975163 \\
\hline $\mathrm{H}$ & -0.52507983071716 & 1.71883588532223 & -0.35702463083159 \\
\hline $\mathrm{C}$ & -0.60970195176701 & 1.68320734674688 & 1.81583794868098 \\
\hline $\mathrm{H}$ & -1.57677240167256 & -2.28745871886048 & 1.42674522138980 \\
\hline $\mathrm{H}$ & -2.15759789196354 & -0.06804943328220 & 0.47579248949766 \\
\hline $\mathrm{H}$ & -1.67305228242650 & 1.43909472528358 & 1.91076277410065 \\
\hline $\mathrm{H}$ & 0.95642408443639 & -0.12855612663043 & -1.52632329173089 \\
\hline $\mathrm{C}$ & 0.93011940061396 & -0.74589524299567 & -0.60468498330858 \\
\hline $\mathrm{H}$ & 0.63788767293482 & -1.75222717858883 & -0.92064672853471 \\
\hline $\mathrm{H}$ & 2.69036913746621 & 0.16270922783420 & 0.32720956540781 \\
\hline $\mathrm{C}$ & 2.32267830728946 & -0.82136497717351 & 0.01783176449544 \\
\hline $\mathrm{H}$ & 2.30656616377115 & -1.46827110225840 & 0.90101428013095 \\
\hline & 3.03997769367837 & -1.22911292959788 & -0.70404835228984 \\
\hline \multicolumn{4}{|c|}{$\mathrm{G}=-183316.0444 \mathrm{kcal} / \mathrm{mol}$} \\
\hline
\end{tabular}

5

Pd 2.62283260277344 -0.73184764453462

C $1.22832055778282 \quad-2.85173618679001$

0.29650498647370

2.17484356412303

C $0.49603198557427 \quad-4.03262478890653$

1.97309323554029

C 0.72707937030987

$-1.91547529082268$

3.09697102986518 


\begin{tabular}{|c|c|c|c|}
\hline $\mathrm{C}$ & -0.67200659719883 & -4.28935736552503 & 2.68999283210872 \\
\hline $\mathrm{H}$ & 0.86016212061930 & -4.76348651710562 & 1.25363352704931 \\
\hline $\mathrm{C}$ & -0.43926991892394 & -2.16798698001833 & 3.80817990316150 \\
\hline $\mathrm{H}$ & 1.24425986514376 & -0.96969362737535 & 3.23064093278999 \\
\hline $\mathrm{C}$ & -1.14218071151162 & -3.36103453557858 & 3.61687366480581 \\
\hline $\mathrm{H}$ & -1.21593395974450 & -5.21572218112544 & 2.52098730732207 \\
\hline $\mathrm{H}$ & -0.81689133030267 & -1.42167647704532 & 4.50212225984183 \\
\hline $\mathrm{H}$ & -2.05780318865984 & -3.55195499788203 & 4.17020571878310 \\
\hline $\mathrm{C}$ & -2.48371617036328 & -1.47664122327666 & 1.10196870423581 \\
\hline $\mathrm{C}$ & -1.39080088147541 & -1.69017889756833 & 0.26659773218043 \\
\hline $\mathrm{C}$ & -2.64674843512896 & -0.25072585422424 & 1.74545660113124 \\
\hline $\mathrm{H}$ & -1.26400366124505 & -2.65351491472790 & -0.21984525993745 \\
\hline $\mathrm{H}$ & -3.49449055382835 & -0.08512157311860 & 2.40506799359820 \\
\hline $\mathrm{C}$ & -0.43411280942138 & -0.685577232 & 492070771 \\
\hline $\mathrm{C}$ & -1.69923003808651 & 0.7573704978 & 8533 \\
\hline $\mathrm{H}$ & -1.80982016011899 & 1.71339947608302 & 2.05519122182561 \\
\hline $\mathrm{C}$ & -0.60678992747667 & 0.54252941486940 & 0.71 \\
\hline $\mathrm{H}$ & 0.13302783563340 & 1.32821777207567 & 0.59129532939883 \\
\hline $\mathrm{H}$ & -3.20041989646849 & -2.27706774070409 & 1.26328927033050 \\
\hline $\mathrm{C}$ & 0.69602221197214 & -0.94680686817626 & -0.85462412041594 \\
\hline $\mathrm{C}$ & 2.492415103 & -2.655 & 1.446860786 \\
\hline $\mathrm{H}$ & 2.69892474351674 & -3.41447027982793 & 0.69209524337015 \\
\hline $\mathrm{C}$ & 1.49361447119975 & 0.0267047501 & -1.4460665397 \\
\hline $\mathrm{H}$ & 2.09248162343598 & -0.227 & -2.3170668126 \\
\hline $\mathrm{H}$ & 0.7266332 & -1.95 & -1.26 \\
\hline $\mathrm{H}$ & 1.27520718636165 & 1.0836364504 & -1.31519176024055 \\
\hline $\mathrm{C}$ & 3.56482514124798 & -1.87920167890431 & 1.89102054714716 \\
\hline $\mathrm{H}$ & & & \\
\hline $\mathrm{H}$ & 3.5074332 & -1.36 & 2.84 \\
\hline $\mathrm{C}$ & 4.3441078 & 0.9416402983 & -0.234 \\
\hline $\mathrm{H}$ & 3.9112333 & 1.39 & 222297055 \\
\hline $\mathrm{C}$ & 3.76143 & 1.21 & 0.97 \\
\hline $\mathrm{H}$ & 4.22921810262163 & 0.93630662835481 & 1.91465649002431 \\
\hline $\mathrm{H}$ & 2.94143132478798 & 1.92310221906554 & 1.03950669762435 \\
\hline $\mathrm{C}$ & 5.57378205817882 & 0.177180463 & -0.469265665 \\
\hline $\mathrm{C}$ & 6.43765342285980 & -0.22309089688160 & 0.56675686849163 \\
\hline $\mathrm{C}$ & 5.90964229127782 & -0.18315690412991 & -1.78685903927772 \\
\hline $\mathrm{C}$ & 7.58009590018788 & -0.96770231761558 & 0.29423719048913 \\
\hline $\mathrm{C}$ & 7.05308083163883 & -0.92909097044142 & -2.06017981087845 \\
\hline $\mathrm{C}$ & 7.89298941082541 & -1.32840502581756 & -1.01963587541957 \\
\hline $\mathrm{H}$ & 6.21416432917796 & 0.05527564687133 & 1.59214527048672 \\
\hline $\mathrm{H}$ & 5.25218024958900 & 0.11934279940581 & -2.59903861123083 \\
\hline $\mathrm{H}$ & 8.23501966751297 & -1.26563190431025 & 1.10919985338300 \\
\hline $\mathrm{H}$ & 7.28885250354939 & -1.20047919244500 & -3.08603086388423 \\
\hline & 8.78687124952082 & -1.90979414199447 & -1.22895418250856 \\
\hline \multicolumn{4}{|c|}{$\mathrm{G}=-662793.5535 \mathrm{kcal} / \mathrm{mol}$} \\
\hline
\end{tabular}

\section{6}

Pd $2.15642637614372 \quad-0.38202994275880 \quad 0.07587594564423$ 

C $\quad 1.62414389764818$
C 1.42278294428494
C 0.68736153167752
C 0.32431759680998
H 2.13626183861428
C -0.40513884645192
H 0.81254247926688
C -0.59609725291811
H 0.18758749365778
H $\quad-1.11821759039897$
H -1.45302708429856
C -2.61549945009947
C -1.50675930377158
C -3.03864972969582
H -1.16781859776346
H $\quad-3.90441455503307$
C -0.79082768303379
C -2.34053962141545
H -2.66635914599619
C -1.23162845613071
H $\quad-0.70126269401987$
H $\quad-3.14441031481192$
C 0.38285888067257
C 2.79864362144967
H 3.58728577674386
C 1.24492618302091
H 1.88924348966403
H 0.45023366590996
H 1.07584173638422
C 3.09077452517020
H 4.10449209014102
H 2.46940272357916
$\mathrm{G}=-468634.6099 \mathrm{kcal} / \mathrm{mol}$
$-2.85099208115122$
$-4.01375739170830$
$-2.55129456705999$
$-4.84056684668609$
$-4.25721970767258$
$-3.38105801188780$
$-1.65802889049169$
$-4.52912061556070$
$-5.73122658901458$
$-3.12660529662951$
$-5.17350433352791$
$-1.18482620199818$
$-1.43462198148437$
0.12543976609904
$-2.45574056200164$
0.32403957743032
$-0.38176219482457$
1.18356949643535
2.20779880569435
0.93455116447701
1.76802293470831
$-2.01685216910830$
$-0.70711087839635$
$-2.00692985592006$
$-2.50411381901988$
0.18531321074080
$-0.15785816658886$
$-1.75868993575384$
1.26003827740064
$-0.77486011967536$
$-0.38381336292990$
$-0.34367293313519$
$\mathrm{N}_{\text {imag }}=0$

7
Pd 1.38882284257124
2.83317621180960
0.11058125613335
$-1.77088728052385$
$-0.44478508014609$
C 0.42818424771755
$-2.16490724217957$
C -0.11454404024920
H 0.91728138343728
$-0.14000766665851$
H -0.06214785570790
C -0.24330235106993
C -0.80562691844114
H -1.29781870742488
C -0.87372831434677
H -1.41171084538667
H 1.02986369187276
C -0.23234231300705
C -0.69950162981427
$-3.19904710485577$
0.52880461200297
$-1.21530685840747$
$-1.51306243905630$
0.11036927722796
0.83534993155268
$-2.50063465995096$
1.92936385760782
3.04934070176451
$-0.78790627397160$
$-1.20586520805786$
0.40370628059378
$-2.12900587665328$
0.73359198780837
$-0.44167695302641$
1.16212364698105
2.08513051185644
0.74479294496051
1.34848664636063
$-1.39399490757329$
$-0.90147139203881$
$-0.19678652943886$

1.70053996013599

0.93490410921164

2.70483180299919

1.15593801485117

0.15048994371724

2.93174437451228

3.30888901018119

2.15883977819823

0.54763972408831

3.71158326108871

2.33615910929603

1.02963015663234

0.22559269044390

1.25308524951623

0.07364987748941

1.87946139797735

$-0.36860538959739$

0.66174609386831

0.82606469042671

$-0.13944894760656$

$-0.59100468031899$

1.48725486909256

$-1.19607634131671$

1.42288562772323

0.85764393932191

$-1.82441369137459$

$-2.62896348169921$

$-1.47506766168956$

$-1.77856495279797$

1.99987490521006

1.93110090853365

2.78024859224102 


$\begin{array}{ccc}\mathrm{H} & -0.92776638648165 & 3.96139256623948 \\ \mathrm{H} & -0.03812453101387 & 2.04499565095439 \\ \mathrm{H} & -1.21018128127880 & 2.94509182280542 \\ \mathrm{~N} & 3.56711389721237 & 2.51712956522886 \\ \mathrm{C} & 3.69183410756917 & 1.75286135931455 \\ \mathrm{H} & 4.76473411266585 & 1.59286630481918 \\ \mathrm{H} & 3.29725398588051 & 2.39416935185088 \\ \mathrm{C} & 2.94115648162055 & 0.42671026202023 \\ \mathrm{H} & 3.38375090155564 & -0.32988785402663 \\ \mathrm{H} & 1.89720485205590 & 0.56901306116771 \\ \mathrm{H} & 2.96130556933918 & 0.03073890923409 \\ \mathrm{C} & 4.22265116123258 & 3.84421633358968 \\ \mathrm{H} & 5.26129825672901 & 3.70129970788397 \\ \mathrm{H} & 4.26814972824383 & 4.29193667147938 \\ \mathrm{C} & 3.47302498558701 & 4.79698564478193 \\ \mathrm{H} & 3.49520989236259 & 4.48216962352916 \\ \mathrm{H} & 2.42230949082527 & 4.87941428234726 \\ \mathrm{H} & 3.92485748577236 & 5.79320091355151 \\ \mathrm{C} & 4.21260719313801 & 1.74526783087399 \\ \mathrm{H} & 5.28366084763488 & 1.61254585570097 \\ \mathrm{H} & 3.75076343037235 & 0.75687612246709 \\ \mathrm{C} & 4.04675367415265 & 2.34960832294658 \\ \mathrm{H} & 4.62627020261795 & 3.26658077729589 \\ \mathrm{H} & 2.98913977073518 & 2.57326541034519 \\ \mathrm{H} & 4.38976916313196 & 1.62619724341212 \\ \mathrm{G}=-457786.5023 \mathrm{kcal} / \mathrm{mol} & \\ & & \\ \mathrm{N} \text { imag } & =0 & \end{array}$

\section{8}

Pd 1.41323090983586

C 0.63230342015635

C 1.25574707458275

C -0.74441341122966

H 2.33042148716589

H $\quad-1.23475810798470$

C 0.52267240113239

C -1.48847129801851

H $\quad-2.56225321128295$

C -0.86531241687626

H -1.45815494628059

H 1.22233861127672

C 1.23474097076261

C 0.66858357336210

H 1.23655885645053

H 2.26212675240439

H $\quad-0.40681292535489$

I 1.90749704560395

C 3.82449642525475

C 4.38439411945290

C 4.46874011000998
2.36143410149521

$-2.53818509022184$

$-1.37783484563810$

$-2.55089799061750$

$-1.37094603876576$

$-3.45396203767132$

$-0.20143955171927$

$-1.39042088280721$

$-1.39082184769428$

$-0.23193030311576$

0.66231490386405

$-3.43407225429240$

0.99855242753950

2.22910137327526

2.93156360877979

0.81241269132688

2.38888246554398

3.18298239591907

4.14240590908273

4.68566574555610

4.20131161099667
$-0.74420114880968$

$-1.96923191474160$

0.75877439706475

0.58315603557283

1.85991405880100

2.07096075370945

2.65019737318761

1.89021063739817

1.23703749346083

1.59342284760388

2.91149408611746

0.73370226763744

1.08121108702469

$-0.26021399601349$

1.65798335715674

2.70477182580866

1.34340863963662

1.60043608084809

$-0.51631114364958$

$-0.27899181242549$

$-0.51293503841074$

$-1.90516006447305$

$-2.04551756661022$

$-2.08983414911917$

$-2.65301124070922$
0.60095103079086

0.08378545319819

$-0.36933556386703$

0.30942663982677

$-0.53873503857629$

0.66315150380647

$-0.60533857744186$

0.07467840628136

0.24592363883354

$-0.37599214148153$

$-0.54380953055537$

0.26092095382031

$-1.07788396093376$

$-1.41453275519627$

$-2.01967252080596$

$-1.39369035501959$

$-1.39829927859894$

3.00874639950706

2.87775428162000

4.03132774767671

1.64505839006402 

C 5.63251895869621
C 5.71531810951036
C 6.29759087868305
H 3.86710151516973
H 4.00263491302175
H 6.08059604997387
H 6.22878938589479
H 7.26772495262657
$\mathrm{G}=-606587.4359 \mathrm{kcal} / \mathrm{mol}$
$\mathrm{N}_{\text {imag }}=0$
5.30743285806673
4.82705412706286
5.37893837930641
4.63000023898558
3.76719843511819
5.73524110603884
4.88016539368880
5.86389865889675

\section{9}

Pd 0.17092190400430

I -0.88925005677045

N 1.09141594372840

C 0.35350968291252

H 0.84919068632168

H -0.64413285190992

C 2.51951501263996

H 2.51870301521655

H 2.96748022140118

C 1.01546419219507

H 1.43903654552480

H 1.66043889401468

C -0.38326444088495

H -0.83519483769472

H -1.04725682540364

H $\quad-0.31287546583829$

C 0.21069008279865

H -0.47931588337964

H -0.20227123968350

H 1.15808970362926

C 3.35704075106561

H 3.55745190989552

H 4.32323566474791

H 2.84251994588017

C -0.66135481501548

C -1.23290256138657

C 0.05535023068519

C -1.08018341242389

C 0.19881249817422

C -0.36658817381055

H $\quad-1.78943474110509$

H $\quad 0.48860855799641$

H $\quad-1.52332617561325$

H 0.75622785804569

$\mathrm{H} \quad-0.25185879995785$

$\mathrm{G}=-595734.6908 \mathrm{kcal} / \mathrm{mol}$
$-0.45242199426038$

$-2.43926142515312$

1.35533642120116

1.70322332786308

2.56702230025796

2.02303049268684

1.07884740286659

0.31496322458499

1.99181050919602

2.49527458068905

3.39466810988440

2.24040569718866

2.78372611191193

1.85737546390213

3.21234374928998

3.50230658280186

0.55326767718616

0.84829003694345

$-0.32482949414978$

0.27298259587395

0.57739448943137

1.35343554930842

0.22328606022972

$-0.25720146617869$

$-4.06960914153120$

$-5.30455740598690$

$-3.86191845614706$

$-6.35614758996758$

$-4.92306123223648$

$-6.16837349573454$

$-5.45165416245494$

$-2.88523968900471$

$-7.32323465598567$

$-4.77098581296799$

$-6.98986564453867$
3.94004638794937

1.57331657114430

2.71555094844779

4.98437265056046

0.76441023581159

4.83318741009929

0.61670258460880

2.65166676642967

$\mathrm{N}_{\text {imag }}=0$

$-1.40179978294430$

$-2.49777485188061$

$-0.48217197408980$

0.76597105865466

1.24485627389801

0.46268133615147

$-0.15169626765167$

0.62733376981430

0.28086543089308

$-1.44027627985440$

$-0.95769872452907$

$-2.28217995818007$

$-1.97185164406452$

$-2.34927815784116$

$-1.21592604084176$

$-2.79584604827437$

1.75549816962985

2.55368056415823

1.24167541547423

2.22472130019800

$-1.32163987476754$

$-2.06572388446176$

$-0.94559338710997$

$-1.81458860405761$

$-1.10977297962064$

$-1.40952197989434$

0.06659049421429

$-0.50233979828049$

0.96310983066881

0.68253898275443

$-2.33075359218612$

0.26668642422264

$-0.72708759038298$

1.88431362990597

1.38451446027519 


\section{TS}

$\begin{array}{lll}\text { Pd } 1.37267496857777 & -2.46606292574217 & 0.38530168972958\end{array}$

I $2.43387190939776 \quad-1.31021888099805 \quad 2.53394588990707$

C $0.38036055981513 \quad-0.65427120185932 \quad 2.46181194474895$

$\begin{array}{llll}\text { C } & -0.58922822866786 & -1.38577412705992 & 3.14636825665485\end{array}$

$\begin{array}{llll}\text { C } & 0.06029437203435 & 0.48240851663176 & 1.72008639895622\end{array}$

C $-1.91664092871097 \quad-0.95665112426728 \quad 3.08500524937863$

H $\quad-0.31991375676763 \quad-2.27556094912157 \quad 3.70494553140976$

C $-1.27338493264859 \quad 0.89397330927183 \quad 1.67059545582596$

$\begin{array}{llll}\mathrm{H} & 0.82900218392067 & 1.02815847238210 & 1.18392558877719\end{array}$

C $\quad-2.26008455733010 \quad 0.17924740749384 \quad 2.35063638053384$

$\mathrm{H} \quad-2.68169223495803 \quad-1.52106309968508 \quad 3.61165692391909$

$\mathrm{H} \quad-1.53533979417938 \quad 1.77581503441162 \quad 1.09183280562700$

H $\quad-3.29564648643684 \quad 0.50449872824555 \quad 2.30521438758870$

C $3.51254607987813 \quad-1.68234632538765 \quad-3.82714847151374$

C $2.48144512171751 \quad-2.14155293060010 \quad-3.01540624916662$

$\begin{array}{llll}\text { C } & 4.75235390556552 & -2.32968948710607 & -3.83453722134835\end{array}$

H $1.52980565185553 \quad-1.61829172159487 \quad-3.01319509634996$

$\mathrm{H} \quad 5.55668398021213 \quad-1.96659227742748 \quad-4.46885779977201$

C $2.65976927154720 \quad-3.26444800544822 \quad-2.18602376005408$

C $4.94483580597874 \quad-3.44615910214903 \quad-3.02048702741326$

H $5.90293119553913 \quad-3.96034663298331 \quad-3.01756902791821$

C $3.91025593716242 \quad-3.90695944971597 \quad-2.20915272165255$

H $4.06676037173356 \quad-4.77569350728996 \quad-1.57303572039905$

H $3.35183575153152 \quad-0.81203929521039 \quad-4.45907451525915$

C $1.60264071605654-3.79321489519570 \quad-1.30649560425592$

H $1.83177479191736 \quad-4.76669933545787 \quad-0.87143390951840$

C $0.27918807485752-3.34437218083959-1.22741026476614$

$\mathrm{H} \quad-0.09413568943309 \quad-2.56272475587117 \quad-1.88563908726715$

$\mathrm{H} \quad-0.47988044716611 \quad-3.99639916342582 \quad-0.80110671040222$

$\mathrm{G}=-606583.9428 \mathrm{kcal} / \mathrm{mol}$

$\mathrm{N}_{\text {imag }}=1$

\section{TS}

Pd $1.26929557424393 \quad-0.85509246337392$

I $0.93953758231300 \quad-3.36304519036875$

N $1.39930962593108 \quad 1.36356370074687$

C $-0.97429937961688-2.49628713282669$

C $\quad-1.95056046363972-2.27225971086050$

C $\quad-1.23691295816220-2.28236449019313$

C $-3.20257545570574-1.79785825945461$

H $\quad-1.73225339257569-2.44508161534703$

C $-2.49383668208551-1.80649798467471$

H $\quad-0.46873388960823 \quad-2.46427057664740$

C $-3.47811246698020-1.56018918220659$

H $-3.96228893323143-1.61103007337231$

H $\quad-2.69889170202951-1.62518497203786$

C $1.33059317291239 \quad 1.96509573514834$

$\mathrm{H} \quad 1.40115035180836 \quad 3.06462143132970$

H $\quad 0.34158417349239 \quad 1.73093840451317$

0.28043499423125

0.36522913277278

0.13747656052776

$-0.23220765940635$

0.74064914975785

$-1.58742677223571$

0.34416771000763

1.78920923231036

$-1.96382897823292$

$-2.33133389923247$

$-1.00368075703791$

1.09934923587142

$-3.01625861696576$

1.49911788836760

1.40868399337015

1.89500825567429 

C 2.68425876645365
H 3.48311987782809
H 2.72592630169469
C 0.25996016606004
H 0.34205795268703
H $\quad 0.38814309403145$
H -4.45363499633162
C -1.11889199619708
H -1.15456742331560
H -1.40549251696123
H -1.86606280674083
C 2.37862817877609
H 2.13311011510822
H 2.37605615159856
H 3.38863613515077
C 2.92282531238184
H 2.27157247006247
H 3.95873910928400
H 2.76142950636337
$\mathrm{G}=-595729.6241 \mathrm{kcal} / \mathrm{mol}$
1.74901259946714
1.43909538772876
2.85069604315794
1.86204653581385
2.96061769857133
1.43801358166229
$-1.18844202342911$
1.47465569718285
0.40264702304465
2.02644237890716
1.68867766058405
1.44361831928909
1.78927699997188
0.34614992042028
1.79339133954276
1.10718618415816
1.51074907409598
1.28902136685517
0.02353342060117
$\mathrm{N}_{\text {imag }}=1$

$-0.51250618211892$

0.16250724539876

$-0.59419898746477$

$-0.68699995416607$

$-0.77914695472146$

$-1.68386746597356$

$-1.30434982090698$

$-0.16863670521698$

0.05011097774185

0.73117417355126

$-0.93989116512019$

2.47446247226388

3.48465390008690

2.46836635940193

2.24269426734495

$-1.87382509841814$

$-2.65435785538780$

$-2.18029501216685$

$-1.80557835790780$

\section{2}

Pd 1.26092558985029

I 2.41372025654695

C -0.28366979732788

C -0.25921760155698

C -1.38416527678407

C -1.37807715561995

H 0.61651297768713

C -2.49528591671949

H -1.38182667870839

C -2.49608885023743

H -1.36220832075516

H -3.35642471073643

H $\quad-3.36036940376270$

C 4.31778443457477

C 3.00428194770596

C 4.98062036151375

H 2.50498559925523

H $\quad 6.00709247186016$

C 2.32552918601817

C 4.31919629377260

H 4.82939548261779

C 3.00764959716479

H 2.49696087515130

H 4.82848272681145

C 0.95637899373803

H 0.52788012580983

C 0.12352239685334
$-0.87220375093775$

0.67915954422495

$-1.23811001626729$

$-2.39272237396852$

$-0.38488434932655$

$-2.70793573768604$

$-3.03442341486677$

$-0.71459888404923$

0.52806330820497

$-1.87413036022008$

$-3.60669679040867$

$-0.05052000218548$

$-2.12149136204103$

$-2.25891370663511$

$-2.00522561018980$

$-3.39594539590471$

$-1.11638654690190$

$-3.59024896165172$

$-2.88455761351328$

$-4.27928660112037$

$-5.16442852352998$

$-4.02397669393295$

$-4.70594706845317$

$-1.56704321818192$

$-2.63830564681685$

$-3.42420160820881$

$-1.60014234287386$
$-0.42206640514351$

1.27683173897137

0.76891260482492

1.54774864457407

0.76909242522497

2.32717574411447

1.56957215152166

1.55311673291185

0.18232902099050

2.32844209184802

2.93923423683363

1.55810416826220

2.93883104681511

$-3.53290154090431$

$-3.15292916488510$

$-3.06405509958888$

$-3.52773817628505$

$-3.36241975905862$

$-2.28630993417816$

$-2.20863833324895$

$-1.83852535999224$

$-1.82158253203728$

$-1.14569889022674$

$-4.19712412492204$

$-1.81889468524942$

$-1.19952231615807$

$-2.21035305683477$ 


$$
\begin{array}{llll}
H & 0.39861510033213 & -0.90859773062598 & -3.00243324280489 \\
H & -0.92631946905524 & -1.62310408093203 & -1.93942367037472 \\
\mathrm{G}=-606591.1289 \mathrm{kcal} / \mathrm{mol} & \\
\mathrm{N}_{\text {imag }}=0
\end{array}
$$

\section{3}

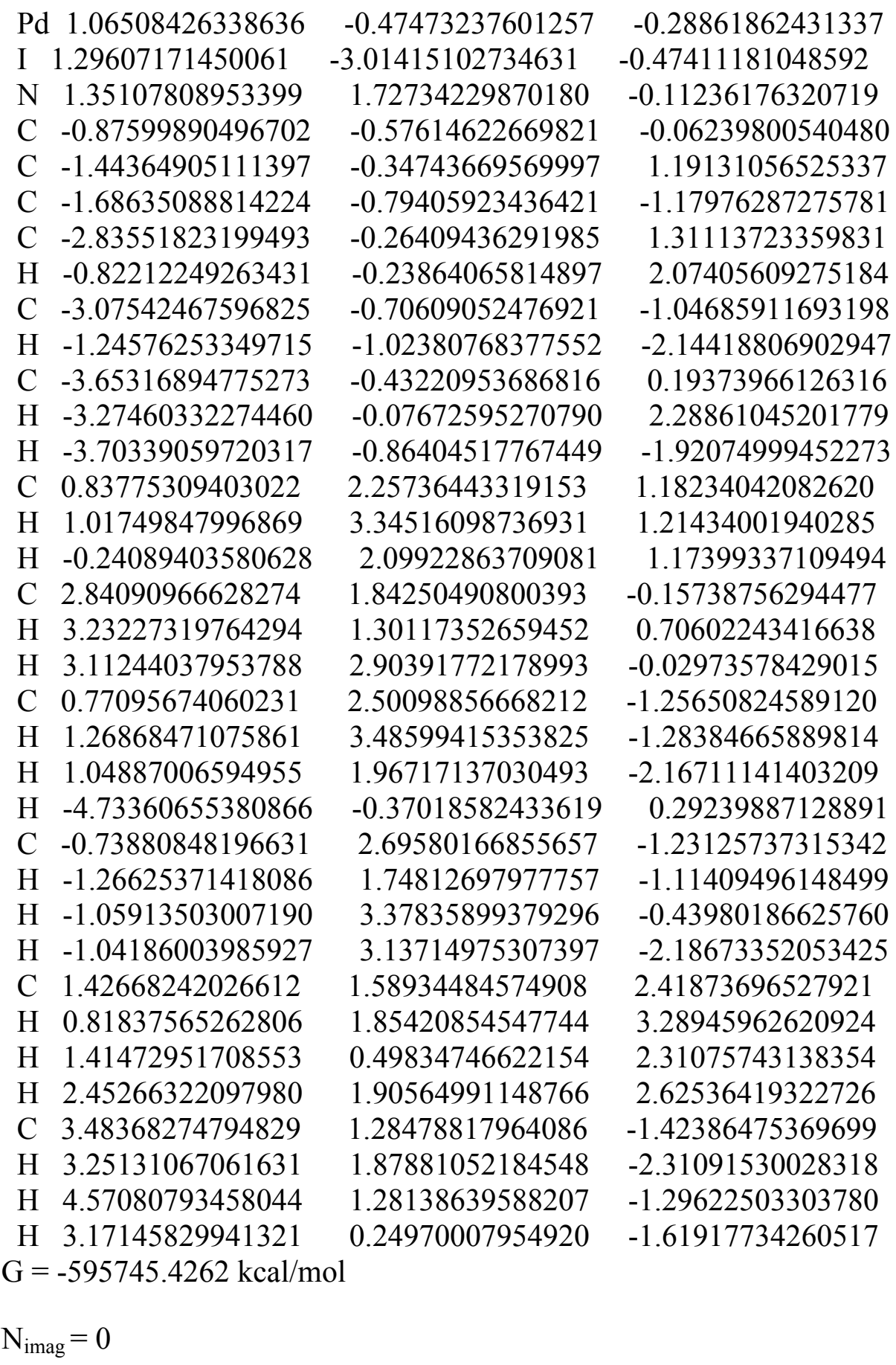

\section{4}

Pd $1.13459686811443 \quad-0.74766644992707 \quad-0.60403234375968$ I $1.36762120152954 \quad 1.67483525518401 \quad-2.00481819465075$

$\begin{array}{llll}\mathrm{N} & 2.76954970146703 & -0.33788660870810 & 1.00804236258825\end{array}$

C $-0.29200994538720 \quad-1.40108019901107 \quad 0.64657552568446$

C $-0.39203321448891 \quad-2.62776457611940 \quad 1.30029088926821$ 


\begin{tabular}{|c|c|c|c|}
\hline $\mathrm{C}$ & -1.28674887594379 & -0.43377159335016 & 0.82371987231520 \\
\hline $\mathrm{C}$ & -1.45515879080571 & -2.86252481369100 & 2.18146600573576 \\
\hline $\mathrm{H}$ & 0.34716086736905 & -3.40649402512405 & 1.15596154033116 \\
\hline $\mathrm{C}$ & -2.34286980206322 & -0.67403994463379 & 1.70702867647739 \\
\hline $\mathrm{H}$ & -1.24348573613646 & 0.50582187847935 & 0.27819681761655 \\
\hline $\mathrm{C}$ & -2.42702016333165 & -1.88688481821348 & 2.39286446564685 \\
\hline $\mathrm{H}$ & -1.51640174007273 & -3.81839158695054 & 2.69730993626893 \\
\hline $\mathrm{H}$ & -3.10324180123061 & 0.09073325977319 & 1.84689605464566 \\
\hline $\mathrm{C}$ & 2.75882747126640 & 1.08755718235596 & 1.44893706678465 \\
\hline $\mathrm{H}$ & 3.63496928107535 & 1.24869583724260 & 2.10062021072308 \\
\hline $\mathrm{H}$ & 2.88184415346061 & 1.69566408782117 & 0.55195994893773 \\
\hline $\mathrm{C}$ & 2.57875824683786 & -1.22148350872493 & 2.19753205465186 \\
\hline $\mathrm{H}$ & 1.54906886593928 & -1.09875298007302 & 2.53101656544764 \\
\hline $\mathrm{H}$ & 3.23795619476277 & -0.85714987805357 & 3.00351563795140 \\
\hline $\mathrm{C}$ & 4.10744098320831 & 20751464265 & 0.4025993447 \\
\hline $\mathrm{H}$ & 4.83283551459929 & -0.71858140328339 & 1.23221951055609 \\
\hline $\mathrm{H}$ & 4.02639235122625 & -1.66275026929115 & -0.02661603126003 \\
\hline $\mathrm{H}$ & -3.25121465223723 & -2.07349624423429 & 3.07608 \\
\hline $\mathrm{C}$ & 4.64467709570386 & 0.28657155755319 & -0.65699629880517 \\
\hline $\mathrm{H}$ & 3.93623131086595 & 0.43351708772623 & -1.47368707573331 \\
\hline $\mathrm{H}$ & 4.90329445708297 & 1.26758514779301 & -0.25078113851154 \\
\hline $\mathrm{H}$ & 5.55749743119332 & -0.15818450396332 & -1.06806372124974 \\
\hline $\mathrm{C}$ & 1.48879986994057 & 1.52716460330613 & 2.15890265784646 \\
\hline $\mathrm{H}$ & 1.55440873220322 & 2.60277216646392 & 2.35258589401604 \\
\hline $\mathrm{H}$ & 0.61451855474320 & 1.35707222386109 & 1.52752147155267 \\
\hline $\mathrm{H}$ & 1.3331766 & 1.02278 & 3.11654 \\
\hline $\mathrm{C}$ & 2.86162595393151 & -2.69785521726524 & 1.94496771779459 \\
\hline $\mathrm{H}$ & 3.93130194823857 & -2.91945437784668 & 1.91369492382386 \\
\hline $\mathrm{H}$ & 3200042465 & -3.29 & 2.7514 \\
\hline $\mathrm{H}$ & 2.42299699323605 & -3.02 & 0.99916 \\
\hline $\mathrm{C}$ & 4.29362136409486 & -2.22589011876620 & -3.42416364635947 \\
\hline $\mathrm{C}$ & 2.9668708 & -1.98514827 & -3.08296 \\
\hline $\mathrm{C}$ & 4.968883 & -3.33 & -2.90652278707891 \\
\hline $\mathrm{H}$ & 2.46648747158512 & -1.10283417152875 & -3.46906108635709 \\
\hline $\mathrm{H}$ & 6.00659942112548 & -3.51500268323292 & -3.17354388531516 \\
\hline $\mathrm{C}$ & 2.28686 & -2.8510 & -2.20291536107733 \\
\hline $\mathrm{C}$ & 4.30196902166641 & -4.21116356095553 & -2.04685402452203 \\
\hline $\mathrm{H}$ & 4.81776345573501 & -5.08006650956343 & -1.64641666201307 \\
\hline $\mathrm{C}$ & 2.97641215246480 & -3.97173614849154 & -1.70055980658573 \\
\hline $\mathrm{H}$ & 2.46021928620090 & -4.65166835008023 & -1.02645026886344 \\
\hline $\mathrm{H}$ & 4.80660841899419 & -1.53834829915122 & -4.09116433884764 \\
\hline $\mathrm{C}$ & 0.92188450603689 & -2.59030956136822 & -1.74194612200401 \\
\hline $\mathrm{H}$ & 0.49363179047423 & -3.36450114472207 & -1.11095955000292 \\
\hline $\mathrm{C}$ & 0.05048221433601 & -1.59946147360643 & -2.24177847696215 \\
\hline $\mathrm{H}$ & 0.29455711214763 & -1.01964752185382 & -3.12432280861981 \\
\hline & -1.00617791560262 & -1.66983490077511 & -2.00249724264102 \\
\hline \multicolumn{4}{|c|}{$\mathrm{G}=-789893.7482 \mathrm{kcal} / \mathrm{mol}$} \\
\hline
\end{tabular}

15TS 
Pd 1.12859495273480

I 0.70779874955351

N 3.28886480460837

C -0.94126295270966

C -1.27282964511345

C -1.88206417888120

C -2.49557417005835

H -0.57697633185278

C -3.11134071379554

H -1.63643312556906

C -3.41709917895189

H -2.73416142537704

H $\quad-3.82409247842819$

C 3.30482200233996

$\mathrm{H} \quad 4.31619179927547$

H 3.13130795345978

C 3.43578271543581

H 2.54262955646524

H 4.29463385663430

C 4.41931614469264

H 5.36488083884158

H 4.33229116332683

H $\quad-4.37441706267754$

C 4.46256471266439

H 3.48683392525882

H 4.76558962154418

H 5.18677581097599

C 2.25931686362753

H 2.20184445914092

H 1.26940411744623

H 2.49625543725576

C 3.62235048214106

$\mathrm{H} \quad 4.54704025908414$

H 3.66264297635756

H 2.78345158009654

C 3.40588350776707

C 2.34309284411702

C 4.53146986069890

H 1.49139866844479

H 5.36011687540750

C 2.38276170622466

C 4.58223292966249

H 5.45013638440201

C 3.52282114799312

H 3.56836908761562

H 3.36162127269101

C 1.28813933800948

H 1.44662192108439

C -0.07410511217027

H -0.31098078483329

H $\quad-0.81606220566192$
$-0.96965495106813$

1.69122004730079

$-0.64563779898600$

$-1.35685916644753$

$-1.85120876702715$

$-0.61152426047142$

$-1.51492353135306$

$-2.49492016387463$

$-0.30218704281140$

$-0.23833555908991$

$-0.73993430352315$

$-1.87647178083686$

0.30286510407914

0.72547463321018

0.90720028791469

1.42719401417357

$-1.62247079120975$

$-1.53339407889393$

$-1.30232410618701$

$-0.79125004625065$

$-0.77404575889600$

$-1.77807477692950$

$-0.48787314465310$

0.25505035459900

0.35700857765286

1.23985627433237

$-0.06563640856378$

0.97085918419279

2.04724125729273

0.65015276592328

0.47488304665033

$-3.08051879100095$

$-3.25934886266621$

$-3.67884919419023$

$-3.43649740725206$

$-1.83423573449302$

$-1.95727599296143$

$-2.65402620660847$

$-1.28961506273588$

$-2.55326694870331$

$-2.90370245553014$

$-3.60298594144928$

$-4.24953446040551$

$-3.72394120522384$

$-4.46481542160145$

$-1.08507273164903$

$-3.06434481454112$

$-3.82879314727324$

$-2.76702500378094$

$-2.35945611324169$

$-3.46059946394035$
0.09768522534769

$-0.47506336903897$

1.22641451304248

0.10305976960927

1.37676100390915

$-0.61989117937643$

1.95841312033777

1.91010895859703

$-0.04087697596052$

$-1.60812112961698$

1.25044142580318

2.95572691253581

$-0.59553510809473$

1.81827989326955

2.22339929186777

1.00389875347379

2.34177252427518

2.96285924954504

2.95898459935134

0.27080565294110

0.84346281534480

$-0.18347240921568$

1.69866519995239

$-0.83444535584539$

$-1.31545676153754$

$-0.46992258734083$

$-1.58967404359936$

2.89856491595417

3.08809540660928

2.55921017845882

3.84459167959387

1.95672698192560

1.40291940430835

2.87348084600502

1.35884915520417

$-3.67126938441589$

$-2.78456988023999$

$-3.54889628808946$

$-2.87638628577417$

$-4.24444416665634$

$-1.73872256087143$

$-2.52618856448891$

$-2.42418660605946$

$-1.63110215055379$

$-0.83719836206132$

$-4.45722472250384$

$-0.78078963315079$

$-0.02355757653227$

$-1.08220439069144$

$-2.05965285165363$

$-0.70651983989348$ 
$\mathrm{G}=-789881.7201 \mathrm{kcal} / \mathrm{mol}$

$\mathrm{N}_{\text {imag }}=1$

16

Pd $1.17094595593851 \quad-1.20016826785224 \quad 0.31666550527074$

$\begin{array}{llll}\text { I } & 2.97481657992999 & -1.98918601788267 & 2.21552158029762\end{array}$

$\begin{array}{llll}\mathrm{N} & 1.44584514470653 & 1.00861989317873 & 0.11050096219446\end{array}$

C $\quad 0.37368901490730 \quad-3.88609590200395 \quad-1.60495113982374$

$\begin{array}{llll}\text { C } & -0.45719641535215 & -4.80910644200082 & -2.24891344217857\end{array}$

$\begin{array}{llll}\text { C } & 1.66364314889596 & -3.66881062703721 & -2.09976700349853\end{array}$

$\begin{array}{llll}\text { C } & -0.00878232126255 & -5.49591546127533 & -3.37739275690100\end{array}$

H $\quad-1.46023499354702 \quad-4.98765337578696 \quad-1.86731476429663$

C $2.11269802798248 \quad-4.35377133520471 \quad-3.22841563470956$

H $\quad 2.31762430159853 \quad-2.96867928296380 \quad-1.58131407792581$

C $1.27665581486389-5.26802330642624 \quad-3.87132724542626$

$\mathrm{H} \quad-0.66335451515280 \quad-6.21088164489615 \quad-3.86933041610510$

H $3.11999159110938 \quad-4.18125821596549 \quad-3.59857983502030$

C $0.25173339182256 \quad 1.65794571876962 \quad 0.73369404936498$

$\mathrm{H} \quad 0.33215266145756 \quad 2.74529210571500 \quad 0.56587965575936$

$\mathrm{H} \quad-0.62528390577310 \quad 1.30422433662580 \quad 0.19255110482603$

$\begin{array}{llll}\text { C } & 2.67904921561497 & 1.41988498364934 & 0.87016638950278\end{array}$

$\begin{array}{llll}\mathrm{H} & 2.60334558637285 & 0.97168917320702 & 1.85931888896528\end{array}$

H $\quad 2.63997343182395 \quad 2.51655904200815 \quad 0.97772640070687$

$\begin{array}{llll}\text { C } & 1.60573889756951 & 1.48179110158574 & -1.30074771864949\end{array}$

$\mathrm{H} \quad 1.90903263307605 \quad 2.54130002636424 \quad-1.25312812749445$

H $2.44003052783632 \quad 0.92311255317149 \quad-1.72610602398018$

H $1.62763655078691 \quad-5.80656984774398 \quad-4.74766344504709$

C $\quad 0.40225591234917 \quad 1.34833445420320 \quad-2.21629750034802$

$\mathrm{H} \quad 0.10079920705432 \quad 0.30641569269007 \quad-2.31901204186890$

$\mathrm{H} \quad-0.45700035816824 \quad 1.93302683855533 \quad-1.87980750576139$

H $\quad 0.68647078331754 \quad 1.71583451861835 \quad-3.20817315449912$

$\begin{array}{llll}\text { C } & 0.07316572145171 & 1.36624793804267 & 2.21756954893429\end{array}$

$\mathrm{H} \quad-0.92684540463643 \quad 1.69269897088058 \quad 2.51891151150063$

$\mathrm{H} \quad 0.15504474169473 \quad 0.29303460037808 \quad 2.41530611949161$

H $\quad 0.80488069487481 \quad 1.88641182520329 \quad 2.84053518974428$

C $4.00027099839886 \quad 1.00663810193422 \quad 0.23867154739633$

$\begin{array}{llll}\mathrm{H} & 4.25410418973007 & 1.59950126439187 & -0.64480090258128\end{array}$

$\begin{array}{llll}\mathrm{H} & 4.79261355854402 & 1.14940537423225 & 0.97974050404228\end{array}$

H $3.99297814658490 \quad-0.05607274060422 \quad-0.01922768906480$

$\begin{array}{llll}\text { C } & -3.13421612793286 & -0.78587429489226 & 1.89896501611896\end{array}$

C $-2.06982705828805 \quad-1.41348514812443 \quad 1.26307488273327$

C $-3.91378844032561 \quad 0.15325292115724 \quad 1.21645014414074$

$\begin{array}{llll}\mathrm{H} & -1.45750405296697 & -2.12016263253271 & 1.81654681518630\end{array}$

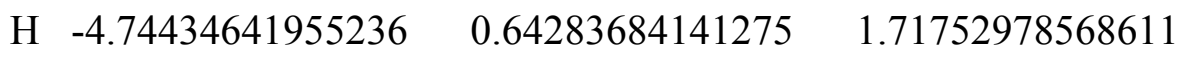

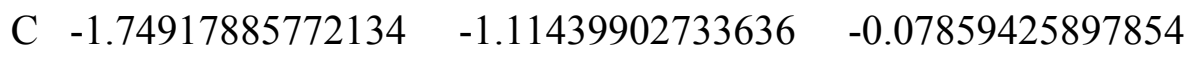

C $-3.62068371328824 \quad 0.45095233701296 \quad-0.11601068818918$

$\mathrm{H} \quad-4.22779412546011 \quad 1.17027157320378 \quad-0.65997639366078$

C $-2.55439153394965 \quad-0.17591145894838 \quad-0.75491843921092$

H $-2.33559250197541 \quad 0.05528460812054 \quad-1.79388911127299$

$\mathrm{H} \quad-3.35471698631985 \quad-1.02356566235687 \quad 2.93640295982585$ 


$$
\begin{array}{lll}
\mathrm{C} & -0.59751272905001 & -1.71347643899264 \\
\mathrm{H} & -0.52729512464049 & -1.47693939005194 \\
\mathrm{C} & -0.13834468095451 & -3.10309234110795 \\
\mathrm{H} & 0.72951341629312 & -3.07812338108761 \\
\mathrm{H} & -0.89291953626876 & -3.65331181723735 \\
\mathrm{G}=-789909.8972 \mathrm{kcal} / \mathrm{mol} & \\
\mathrm{N}_{\text {imag }}=0
\end{array}
$$

\section{TS}

Pd $1.07130744703540 \quad-1.30137192438085$ I $2.80621800198224-2.16578984450447$

N $1.49211302207653 \quad 0.94539915184168$

C $0.34608638925976-3.72697415865818$

C 0.01272737991020

C 1.21527839884526

C 0.51270707707492

H -0.64914475624168

C 1.71527799816215

H 1.52317320482191

C 1.36344538712877

H $\quad 0.23966566202356$

H 2.39517493505312

C 0.38543878101678

H 0.51970127836001

H $\quad-0.54592686147742$

C 2.79167702898150

H 2.74955660324213

H 2.85460131544031

C 1.58748020313286

H 1.99128981943658

H 2.33022178022494

H $\quad 1.76259475344185$

C 0.30741696808246

$\mathrm{H}-0.12511427852841$

H -0.44878539453793

H 0.55193811806316

C 0.27372899111065

H -0.67260557170815

H $\quad 0.27325728282488$

H 1.08432654041609

C 4.03163152223056

H 4.27451150180074

H 4.88333574389241

H 3.91893821184899

C -3.14261002082729

C -2.12915703942086

C -3.93435713223696

H -1.50031891058796

H $\quad-4.72108667805832$

C -1.87906864712539
$-5.08535897835222$

$-3.18463715509676$

$-5.87738500834954$

$-5.52189999389156$

$-3.97399194927966$

$-2.14568487999023$

$-5.32385913404205$

$-6.92798074507337$

$-3.54073264213528$

1.62673497696767

2.71772410632799

1.36129727160107

1.23813105295626

0.76241992428359

2.32980718872468

1.49874826444661

2.52293381204557

0.89797296328335

$-5.94107931591519$

1.53278727170958

0.53875641724594

2.20229606559112

1.89457477269253

1.24615563826227

1.63354583997756

0.15866401779115

1.65648213108490

0.74040000464417

1.33647230053626

0.79478910982574

$-0.30864565193328$

$-0.71861810498501$

$-1.34304745683367$

0.26604114671944

$-2.07739483913520$

0.75703338506034

$-1.00186308030643$
$-0.75253906130727$

$-1.81236157241579$

$-0.42474427756220$

0.35249808519958

0.14769415288955
0.21314706292580

2.12553080533663

0.11580182499226

$-1.68290476914269$

$-1.77017296296260$

$-2.64149401389088$

$-2.80385308475230$

$-1.02496347807764$

$-3.67231606561538$

$-2.55058042507896$

$-3.76033547529914$

$-2.85793108388132$

$-4.40192095174386$

0.85380677128458

0.75358400711299

0.35242024274340

0.80311423344660

1.78174176374664

0.95143768611051

$-1.27080848446148$

$-1.19586217329815$

$-1.79840045631073$

$-4.56084768042419$

$-2.08936270651549$

$-2.18384769166716$

$-1.67170509199583$

$-3.09389666937935$

2.32423703978054

2.71468200614621

2.44487104068292

2.93132822913697

0.07246008238925

$-0.81197637360392$

0.75830219466782

$-0.21691257716619$

1.97099515934066

1.25334672863460

1.37151743614986

1.74881054220093

1.93780418222849

$-0.09164043441984$ 


$$
\begin{array}{ccr}
\mathrm{C} & -3.70430776476232 & 0.61092142167937 \\
\mathrm{H} & -4.31694368309304 & 1.37004983497805 \\
\mathrm{C} & -2.69256696169113 & -0.01793378478759 \\
\mathrm{H} & -2.52444849446301 & 0.25293797681083 \\
\mathrm{H} & -3.30976296251045 & -0.99303808300187 \\
\mathrm{C} & -0.80146722971661 & -1.61557886133669 \\
\mathrm{H} & -0.73061796101148 & -1.28697660193837 \\
\mathrm{C} & -0.25217203805663 & -2.90310592592297 \\
\mathrm{H} & 1.00833874279683 & -2.82936651385252 \\
\mathrm{H} & -0.75946470366252 & -3.49071141338476 \\
\mathrm{G}=-789906.1182 \mathrm{kcal} / \mathrm{mol} \\
\multicolumn{3}{l}{} \\
\mathrm{N}_{\text {imag }}=1
\end{array}
$$

\section{8}

Pd 0.71148789864689 I 2.39815495222255 N 0.93118327991995 C -1.70705265512264

C -2.96691831341936

C -1.34970548595936

C -3.83859807384848

H -3.25225479226203

C -2.22196886120938

H -0.37283622981144

C -3.46756034284762

H $\quad-4.80641962757169$

H $\quad-1.92325698171337$

C -0.19227897921841

H -0.14017052790622

H -1.11754768872080

C 2.24398642336210

H 2.30061098112067

H 2.22848747240864

C 0.84659869434944

H 1.18449897691754

H 1.57074483570754

H -4.14305346930890

C -0.51361050639475

H -0.93856622281149

H -1.23271164658851

H -0.38082918995467

C -0.22974296914496

H -1.13628708309359

H $\quad-0.26800275249747$

H 0.62857938581431

C 3.45899809687503

H 3.57855721859523

H 4.35513305721295

H 3.40675305389393

C 1.48774266665572

$-1.43342780673306$

$-1.73619223037736$

0.77340269974051

$-2.63767915204175$

$-2.09387800775118$

$-2.76768667098357$

$-1.68338699876326$

$-1.98415128600881$

$-2.35694515020027$

$-3.15400309124799$

$-1.81082937091171$

$-1.26305358194171$

$-2.45600868128014$

1.41691873077702

2.50244869192156

1.04656120552035

1.26138933716294

0.95005248868023

2.36314986970531

1.17844553689875

2.22543731613295

0.56552918666963

$-1.48800851123362$

1.06293413849465

0.06148375042174

1.79826041990184

1.23966718486184

1.15106553328047

1.61431969091148

0.08044205418036

1.57086106864426

0.72026549804644

1.14972951008809

0.95981318273369

$-0.37040818750752$

$-4.95264304086302$
0.03915945211692

$-0.44111396769655$

$-0.68373148089819$

$-1.72244361421982$

3.00922946020779

$-0.87486881914036$

$-1.90760568753304$

$-0.60117101672696$

0.48508603867908

0.16326524584062
0.42011329370427

2.64900701532835

0.14228423393226

1.57461022088838

1.26502571058345

2.92891680112591

2.26961407997827

0.22069605037931

3.93109109608706

3.19920486134804

3.60942976104807

2.00817679805771

4.97129976016549

0.89784850435589

0.70681536202471

0.45472161532281

0.67314657872711

1.71512462259309

0.63523934702619

$-1.30418915384953$

$-1.36778636735354$

$-1.84106492012582$

4.39723607680753

$-1.97688308394674$

$-1.85064662453020$

$-1.60686597738154$

$-3.04962795082860$

2.39523108063226

2.80003217101668

2.60928056746973

2.92431696478756

$-0.06614805961657$

$-1.06485571339528$

0.51447652880410

$-0.14507137997939$

$-2.82668240687522$ 

C $\quad 0.72613197871169$
C 2.73078378408186
H 3.32415207826476
C 1.18865594960954
C 3.20356600738850
H 4.17096876918222
C 2.44066025192847
H 2.81719307655079
H 1.11166578156577
C 0.43955362851401
H 0.80625614014691
C -0.82008763301394
H -0.04360904010361
$\mathrm{H}-1.31728329403799$
$\mathrm{G}=-789908.5698 \mathrm{kcal} / \mathrm{mol}$
$-4.33318658068604$
$-5.50988495814382$
$-3.90044140963198$
$-5.99137685014331$
$-4.26105011044089$
$-5.44271058863431$
$-5.86830517176651$
$-4.82591856161101$
$-4.74905038239122$
$-5.00303116627831$
$-3.62160094177654$
$-3.86140968302694$
$-3.01594574277607$
$-1.34706998353981$
$-3.04203444408235$
$\mathrm{N}_{\text {imag }}=0$

\section{9}

Pd 0.91600476154377

I 2.22123235470563

$-1.16473999075981$

$-2.56219271503210$

N 1.38244302173452

1.07579875250059

C 0.49267604815628

1.70232877813918

H $\quad 0.67414851745947$

2.79191896947364

H $\quad-0.53571963529838$

C 2.80366562837420

1.55068883496052

1.39331039255758

H 2.99119171721268

H 2.93683475782884

1.03168711513438

2.48956157828121

1.52220883815877

C 0.96785303005734

H 0.72408062474452

2.59730892541525

1.38130045316790

H 1.81082778954903

0.69490407010884

C -0.20985919059248

$-0.39651663395999$

H 0.04547836536489

H $\quad-1.11413733026698$

H $\quad-0.45224595124236$

0.81501835502956

0.95606401172277

C 0.63091253375673

1.14038456255154

H -0.14345277061888

H 0.50529327182732

1.58293955876711

0.05400626561030

H 1.59936942282721

1.37111668406404

0.73698123832189

C 3.81234255090196

H 3.80079073143338

H 4.81853660635937

1.15796219988603

0.88953045399026

H 3.63238097177702

H $\quad 0.51167149540490$

$-0.34075610939837$

$-2.60621587969112$

$\mathrm{G}=-450862.8906 \mathrm{kcal} / \mathrm{mol}$

$\mathrm{N}_{\text {imag }}=0$

20

Pd $1.33880894395314 \quad-1.51853110700751$

I $3.40734915782699-1.63312955096874$

0.71801745589025

2.35756870600868

$-1.84151049268453$

$-2.51274212624031$

$-2.10709655264362$

$-3.28558090179451$

$-0.51553400874781$

$-1.20126286182736$

$-0.94742505142592$

$-0.21356911976554$

0.80315853869593

$-3.84553497086676$

0.57464316742436

1.56676957990808

0.46406690732951

$-0.91601772839211$

$-0.50130382628124$

0.08850993478306

1.77566152354930

0.32809481130919

1.34504830276568

1.34666895483962

1.00492082283852

0.62378022864662

1.63655730251693

0.63062511763368

$-1.02636265559412$

$-1.02477694008460$

$-1.70349213758509$

$-1.53568137180061$

$-1.58714009606974$

$-0.93521121462656$

$-2.57149699068294$

2.75360446104501

3.38941579330863

2.75413247949901

3.20502180498641

$-0.31048227811255$

$-1.32043811444627$

0.09354593775316

$-0.37290595524030$

$-0.19756149923205$ 


\begin{tabular}{|c|c|c|c|}
\hline $\mathrm{N}$ & 1.22647088691769 & 0.99387057007841 & 0.34029037041218 \\
\hline $\mathrm{C}$ & 0.42813803212547 & 1.61634411290350 & 1.43135182128916 \\
\hline $\mathrm{H}$ & 0.42940717173266 & 2.71304807668815 & 1.28837992437753 \\
\hline $\mathrm{H}$ & -0.60206937551298 & 1.27669021149621 & 1.30639045909378 \\
\hline $\mathrm{C}$ & 2.64145112331257 & 1.44535376692139 & 0.43844037288424 \\
\hline $\mathrm{H}$ & 3.02707871672600 & 1.10514525620046 & .39967158334613 \\
\hline $\mathrm{H}$ & 2.65660817077487 & 2.55077999541551 & 0.43674384734005 \\
\hline $\mathrm{C}$ & 0.69579577565222 & 1.43877510540113 & -0.98129376326094 \\
\hline $\mathrm{H}$ & 1.04937884716686 & 2.46875979197203 & -1.16840871363433 \\
\hline $\mathrm{H}$ & 1.14536539043463 & 0.80104190645494 & -1.74221926942955 \\
\hline $\mathrm{C}$ & -0.81906049151358 & 1.42434334351614 & -1.14167215415189 \\
\hline $\mathrm{H}$ & -1.25466186262996 & 0.47193093393890 & -0.83484050450350 \\
\hline $\mathrm{H}$ & -1.30800408197403 & 2.22110076037419 & -0.57507415189010 \\
\hline $\mathrm{H}$ & -1.05747285435601 & 1.57180 & 4335 \\
\hline $\mathrm{C}$ & 0.86763469505161 & 1.28776155016886 & 4385764 \\
\hline $\mathrm{H}$ & 0.15412569074560 & 1.74290005463033 & 62987832 \\
\hline $\mathrm{H}$ & 0.8792145793 & 0.20861307 & 7793 \\
\hline $\mathrm{H}$ & 1.8605 & 1.67 & 607 \\
\hline $\mathrm{C}$ & 3.5519 & 0.91177 & 7467 \\
\hline $\mathrm{H}$ & 893166975 & 1.31768 & 3136 \\
\hline $\mathrm{H}$ & 4.58 & & 576 \\
\hline $\mathrm{H}$ & 3.4975 & -0.1 & 353 \\
\hline $\mathrm{H}$ & 77693227 & -2.9439 & 2292 \\
\hline $\mathrm{C}$ & 94279841 & -2.5618 & 0495 \\
\hline $\mathrm{C}$ & 1977 & 593 & 454 \\
\hline $\mathrm{H}$ & 0.931 & -3.5 & 2745 \\
\hline $\mathrm{H}$ & 952915245 & -2.999 & 9462 \\
\hline $\mathrm{H}$ & -1.2685 & -1.423 & 9791 \\
\hline $\mathrm{C}$ & 0.7493 & -1.8 & 6918 \\
\hline $\mathrm{C}$ & 29023618 & -1.7896 & 5017 \\
\hline $\mathrm{C}$ & 338640283 & -1.1670 & 35864 \\
\hline $\mathrm{C}$ & 2.4225 & -1.07 & 5834 \\
\hline $\mathrm{C}$ & 0.107 & -0.4 & 7468 \\
\hline $\mathrm{C}$ & 1.43785208528708 & -0.40 & 0664 \\
\hline $\mathrm{H}$ & 2.84629687155640 & -2.29875 & 1746 \\
\hline $\mathrm{H}$ & -1.27 & & 0190 \\
\hline $\mathrm{H}$ & 3.45946107071219 & -1.0405 & 2139 \\
\hline $\mathrm{H}$ & -0.66644263272619 & & 42804 \\
\hline $\mathrm{H}$ & 1.70462856685954 & 0.15 & 2303918 \\
\hline \multicolumn{4}{|c|}{$\mathrm{G}=-645022.5108 \mathrm{kcal} / \mathrm{mol}$} \\
\hline \multicolumn{4}{|c|}{$\mathrm{N}_{\text {imag }}=0$} \\
\hline \multicolumn{4}{|c|}{ 21TS } \\
\hline & 0041 & -0.7 & 0639 \\
\hline & 3.73132843946409 & -1.54415515050286 & 0.73040382307402 \\
\hline $\mathrm{N}$ & 0.85821688775437 & 1.47586465007339 & 0.58008062354761 \\
\hline $\mathrm{C}$ & 0.53588283942223 & & \\
\hline $\mathrm{H}$ & 356751287 & 2.71440057744112 & 2.26053244691249 \\
\hline $\mathrm{H}$ & -0.46861783607480 & 1.23479041415528 & 2.17101200310829 \\
\hline $\mathrm{C}$ & 2.20103474515166 & 2.05854840033312 & 0.29347583530345 \\
\hline
\end{tabular}




$\begin{array}{clcc}\mathrm{H} & 2.92002494109884 & 1.51349446840081 & 0.90614484491413 \\ \mathrm{H} & 2.20473597449533 & 3.11305261259782 & 0.62198140614544 \\ \mathrm{C} & -0.16561464258808 & 2.18464279669073 & -0.24168922692551 \\ \mathrm{H} & -0.04499084133352 & 3.27318597394396 & -0.09460174453829 \\ \mathrm{H} & 0.06707821322079 & 1.97240349633925 & -1.28699231413054 \\ \mathrm{C} & -1.61067016390638 & 1.78804083861536 & 0.03858236407185 \\ \mathrm{H} & -1.71274018349701 & 0.69996075922106 & 0.08269117424038 \\ \mathrm{H} & -1.98912205970111 & 2.21214112454659 & 0.97237957565732 \\ \mathrm{H} & -2.24606480767865 & 2.15945828776138 & -0.77238528526528 \\ \mathrm{C} & 1.48020896358339 & 0.92182954518788 & 2.98214400816102 \\ \mathrm{H} & 1.08198705881101 & 1.01052008404353 & 3.99878995214716 \\ \mathrm{H} & 1.55790127536472 & -0.14077182721930 & 2.73408118694482 \\ \mathrm{H} & 2.48827404815562 & 1.34337763071901 & 2.98284620768475 \\ \mathrm{C} & 2.63648309917638 & 1.96072159044329 & -1.16404864677139 \\ \mathrm{H} & 2.07786390864728 & 2.63304977958325 & -1.82129995185485 \\ \mathrm{H} & 3.69567109216841 & 2.22799051223729 & -1.23486714010392 \\ \mathrm{H} & 2.52935365029284 & 0.93501671984560 & -1.53577959344031 \\ \mathrm{H} & 1.89178951694135 & -1.93624072316168 & 0.45643848562618 \\ \mathrm{C} & 0.31595028580825 & -2.40198459412849 & -1.32993999272812 \\ \mathrm{C} & -0.81481203013113 & -1.66666134847110 & -0.94073392277400 \\ \mathrm{H} & 0.51249245379669 & -3.33990612330961 & -0.81374183510905 \\ \mathrm{H} & -1.46312096164383 & -2.06027608820371 & -0.16239176472589 \\ \mathrm{H} & -1.28190320158386 & -0.95345175513471 & -1.61537736271387 \\ \mathrm{C} & 1.07403469108901 & -2.22091916593843 & -2.58259172235358 \\ \mathrm{C} & 2.19826489489378 & -3.03000136628586 & -2.82054228923412 \\ \mathrm{C} & 0.74646278189594 & -1.24442135612994 & -3.54069612038961 \\ \mathrm{C} & 2.97198505231570 & -2.86622204593741 & -3.96630954877332 \\ \mathrm{C} & 1.51863633604843 & -1.08158558701649 & -4.68669936075412 \\ \mathrm{C} & 2.63753724495479 & -1.88967233552410 & -4.90556596165880 \\ \mathrm{H} & 2.47684141948943 & -3.77589990042275 & -2.08035525695127 \\ \mathrm{H} & -0.11802677475692 & -0.60480307623232 & -3.38670984501261 \\ \mathrm{H} & 3.84242960435042 & -3.49815400160174 & -4.12285012887213 \\ \mathrm{H} & 1.24714038409436 & -0.32082056238465 & -5.41465216253207 \\ \mathrm{H} & 3.24122563926980 & -1.75852035527457 & -5.79963469454036 \\ \mathrm{G}=-645020.4762 \mathrm{kcal} / \mathrm{mol} & & \\ \mathrm{N} \mathrm{imag} & =1 & & \\ & & & \end{array}$

22

Pd 0.97391325539775

I 3.24675905031063

N 1.13011256506086

C 0.11368295574418

H $\quad 0.29216850444010$

H $\quad-0.86330968562248$

C 2.49398682697356

H 2.60726698311192

H 2.53606312502202

C 0.87368657681719

H 0.85980732095446

H $\quad 1.72607571730977$
$-0.83008172931282$ $-1.63786585836753$

1.40853972101671

1.87874833566746

2.94859631240185

1.79871169648825

1.78585601389220

1.35595568368093

2.88392674764499

2.04047109568157

3.13641387333227

1.79607352836081
0.19163345598739 2.82090734885238

0.42915666981007

1.41637227250923

1.61705067428862

0.93558154205986

0.91018069240160

1.90637286050058

1.00847359581174

$-0.89958981119923$

$-0.77100443112018$

$-1.53461826236707$ 


$\begin{array}{clc}\mathrm{C} & -0.39403455328935 & 1.57035223369307 \\ \mathrm{H} & -0.39232942916640 & 0.48071895178442 \\ \mathrm{H} & -1.30888927614658 & 1.86333875968074 \\ \mathrm{H} & -0.42951946892395 & 2.01014905101308 \\ \mathrm{C} & 0.08456947622604 & 1.08693975250206 \\ \mathrm{H} & -0.71178263642664 & 1.48031408544423 \\ \mathrm{H} & -0.12445969551190 & 0.02811491767467 \\ \mathrm{H} & 1.02328874430568 & 1.14039509898116 \\ \mathrm{C} & 3.63900414464143 & 1.28699267246757 \\ \mathrm{H} & 3.71578708306230 & 1.81890369282501 \\ \mathrm{H} & 4.58016356988455 & 1.42897424764339 \\ \mathrm{H} & 3.52826322394735 & 0.21587050990406 \\ \mathrm{H} & 2.04524838775033 & -1.23291572941243 \\ \mathrm{C} & 1.48919720883705 & -2.51191695332889 \\ \mathrm{C} & 0.59117042592114 & -2.95048951336846 \\ \mathrm{H} & 2.55157930607270 & -2.67801684530761 \\ \mathrm{H} & 0.96111818229290 & -3.50070169736219 \\ \mathrm{H} & -0.46993288201298 & -3.05834489679837 \\ \mathrm{C} & 1.15211450191708 & -2.04105380895229 \\ \mathrm{C} & 2.15168998557633 & -1.42007082252327 \\ \mathrm{C} & -0.13783685371660 & -2.15024854786233 \\ \mathrm{C} & 1.86773673169568 & -0.90158331319294 \\ \mathrm{C} & -0.42100694426293 & -1.63268250213865 \\ \mathrm{C} & 0.57685787219870 & -0.99993405618365 \\ \mathrm{H} & 3.15447323862626 & -1.33172342662087 \\ \mathrm{H} & -0.92356602182282 & -2.64329739990678 \\ \mathrm{H} & 2.65469365883366 & -0.42104943479217 \\ \mathrm{H} & -1.42476000476767 & -1.72763634854847 \\ \mathrm{H} & 0.35267438173859 & -0.59743399480083 \\ \mathrm{G}=-645024.5046 \mathrm{kcal} / \mathrm{mol} \\ & & \\ \mathrm{N} \text { imag } & =0 & - \\ & & \\ & & \\ & \end{array}$

\section{3-p-OMe}

Pd 1.08621433600439

I 1.32042793608261

N 1.36727370064257

C -0.84462782212596

C -1.36230237969783

C -1.71226654441704

C -2.74929460215460

H -0.70745601650976

C -3.08918658278322

H -1.32077065848102

C -3.61746877443544

H -3.12661070087269

H $\quad-3.77281173252394$

C 0.87920914929603

H 1.04580193306202

H $\quad-0.19709928060438$

C 2.85442886237550
$-0.43654012424860$

$-2.96692204658072$

1.76399015308810

$-0.56161318258692$

$-0.37162648714859$

$-0.77012757091990$

$-0.31020798954345$

$-0.27404090880743$

$-0.70618069768737$

$-0.97630492947263$

$-0.46701752148119$

$-0.15353908969875$

$-0.85513161770988$

2.26345532661072

3.35291810237141

2.09009984791719

1.88682913777007
$-1.60184077799978$

$-1.72017752334263$

$-1.07941892125298$

$-2.60424547481280$

2.71668766408581

3.35802101549378

2.52175362061442

3.27237815155325

0.03881318904095

$-0.91391371255414$

0.57889015743853

$-0.15919140886481$

1.46222895853984

$-1.07842930929866$

$-0.10555428193650$

$-0.90175477933255$

0.75387451976508

$-0.31865002147590$

$-2.43394580141930$

$-3.20614545133757$

$-2.98412952348512$

$-4.46602074036302$

$-4.24472192337793$

$-4.99043606454608$

$-2.79421819198092$

$-2.41930085252147$

$-5.04195173144888$

$-4.65141121927190$

$-5.97458024844365$
$-0.34092460444872$

$-0.59999800658543$

$-0.12002423607521$

$-0.02282007571439$

1.25372179566933

$-1.10154407673238$

1.45381483654869

2.11336658670513

$-0.90793502647312$

$-2.09211744340151$

0.36876576157125

2.45848923328536

$-1.73927422544086$

1.19601831752042

1.24355096138410

1.21101345898326

$-0.19627853609239$ 


$\begin{array}{clc}\text { H } & 3.26637492496549 & 1.32882874515996 \\ \mathrm{H} & 3.12619762178040 & 2.94653618868860 \\ \mathrm{C} & 0.75664396007304 & 2.55776073823258 \\ \mathrm{H} & 1.23663993306711 & 3.55214872496615 \\ \mathrm{H} & 1.02924015756279 & 2.05407685791906 \\ \mathrm{C} & -0.75585482514780 & 2.72206959337438 \\ \mathrm{H} & -1.26043231478365 & 1.76052350878503 \\ \mathrm{H} & -1.07572377925318 & 3.37925888748437 \\ \mathrm{H} & -1.08565025629193 & 3.17844963924641 \\ \mathrm{C} & 1.50697251318036 & 1.58117270180446 \\ \mathrm{H} & 0.91530307487592 & 1.82032746963739 \\ \mathrm{H} & 1.50866463234083 & 0.49251028193832 \\ \mathrm{H} & 2.53275882410753 & 1.90901881447174 \\ \mathrm{C} & 3.47013175682845 & 1.35550561370932 \\ \mathrm{H} & 3.22448279208185 & 1.97099581119980 \\ \mathrm{H} & 4.55945234202315 & 1.34257989066935 \\ \mathrm{H} & 3.14831083392244 & 0.32675481204016 \\ \mathrm{O} & -4.98459298902045 & -0.42056220850794 \\ \mathrm{C} & -5.56420259021847 & -0.22319892018989 \\ \mathrm{H} & -5.29178891712636 & -1.03261770726838 \\ \mathrm{H} & -5.26076862643176 & 0.74029559193285 \\ \mathrm{H} & -6.64485952039301 & -0.22656669216579 \\ \mathrm{G}=-667582.2378 \mathrm{kcal} / \mathrm{mol} & \\ & & \\ \mathrm{N} \text { imag } & =0 & \end{array}$

\section{3-p-OCF 3}

Pd 1.17481857770345

I 1.48707844505728

N 1.37225769857342

C -0.69193905458428

C -1.06103626134365

C -1.66528938146778

C -2.41376480736678

$\mathrm{H} \quad-0.31228611479195$

C -3.01736001044797

H -1.37991715644823

C -3.37266481596678

H -2.71998632906815

H $\quad-3.78695299099667$

C 1.04030725072438

H 1.16705034210216

H $\quad-0.01751348923068$

C 2.83068581040777

H 3.37438466059993

H 3.06862149530061

C 0.58612439094735

H 1.02216051670348

H $\quad 0.75259745410480$

C -0.91045840169430

H -1.36444334578014
$-0.40283813920751$

1.76930289476611

$-0.67844159638647$

$-0.65584437820105$

$-0.81253341188084$

$-0.67923438611736$

$-0.62255697218057$

$-0.83383685515717$

$-0.89263491046164$

$-0.74685436894838$

$-0.65192986120496$

$-0.92380660687106$

2.08957193105159

3.17414903493109

1.85928179568943

1.98891537125511

1.36603201438284

3.04012924680095

2.64674349833293

3.65956225079913

2.26379578110472

2.72454071530805

1.73384657137908
$-2.87421636025446$
0.64650385674015

$-0.05463940496912$

$-1.23306934234080$

$-1.24031862111296$

$-2.16220257130347$

$-1.17825519117703$

$-1.07226021798850$

$-0.36528217730577$

$-2.11758297201665$

2.40532079868643

3.29480414415813

2.27629262794540

2.59425627028020

$-1.48765833368061$

$-2.35636444943188$

$-1.38145902458287$

$-1.70118715153846$

0.45097131877818

1.73544057511057

2.42692146675116

2.16919130214085

1.57944681415350

$-0.51832569074124$

$-1.08576427601249$

$-0.07950272293026$

$-0.01071086312021$

1.33567285827317

$-1.00616115478834$

1.68731245549230

2.11961318763933

$-0.65703739721692$

$-2.04934891973158$

0.68421037177617

2.72839781341588

$-1.41711701641938$

1.33818168563779

1.49226982333491

1.46661129955500

$-0.32542137475319$

0.38732105921467

$-0.09204389063880$

$-1.00607157124444$

$-0.95916265694711$

$-2.01448382772372$

$-0.73906625462447$

$-0.69454410053470$ 


$\begin{array}{cll}H & -1.14531151178388 & 3.26123473723616 \\ H & -1.37631268835590 & 3.27333701041930 \\ C & 1.84812694085347 & 1.31255021994835 \\ H & 1.36835927070155 & 1.41636083848382 \\ H & 1.87986372888534 & 0.24681863877567 \\ H & 2.87505242995165 & 1.67474841340140 \\ C & 3.29611402955264 & 1.64599929310932 \\ H & 2.90451274731421 & 2.33485999422695 \\ H & 4.38892678910420 & 1.69680760484252 \\ H & 3.00575178846966 & 0.62715135533439 \\ O & -4.74019264641097 & -0.82450776127836 \\ C & -5.42112058297419 & 0.34166993809966 \\ F & -5.45337032562604 & 1.02976446961342 \\ F & -6.68145557871331 & 0.05417217782338 \\ F & -4.90204039100568 & 1.18808241803444 \\ G=-854468.2776 \mathrm{kcal} / \mathrm{mol} \\ \mathrm{N}_{\text {imag }}=0\end{array}$

\section{TS-p-OMe}

Pd 1.15141787237929

I 0.72183003282939

N 3.33842419948613

C -0.91468107129019

C -1.35188178795863

C -1.80569363019676

C -2.60970937084423

H -0.69984435030591

C -3.08005221461972

H -1.49419713810743

C -3.48235970429392

H -2.94398692417013

H $\quad-3.73383573467841$

C 3.36009060912126

H 4.38157652588271

H 3.15747403461483

C 3.53266585569086

$\mathrm{H} \quad 2.63589497310804$

H 4.37385457563275

C 4.44663133524576

H 5.40672939953524

H 4.37113749006828

C 4.43521144858811

H 3.44653317522758

H 4.71789972063019

H 5.15069470812345

C 2.34616355402521

H 2.28416273743660

H 1.34931619749228

H 2.61702083415068

C 3.79986059902217
$-0.98846911143642$ 1.68599080749533

$-0.67967929554677$

$-1.31129477454616$

$-1.72399197483236$

$-0.61758156153707$

$-1.36928985545271$

$-2.32261679630703$

$-0.27662369629583$

$-0.30064368655792$

$-0.63540211643365$

$-1.66265999008095$

0.28900898683789

0.68051578620564

0.86235213444946

1.39721528032933

$-1.66825643302004$

$-1.63220121009933$

$-1.31483066100531$

$-0.79303291798825$

$-0.75497939621035$

$-1.77913528807450$

0.25793229948299

0.33396614287648

1.25001340128800

$-0.03991371152479$

0.89411804408929

1.96586028952418

0.56999788676122

0.38117723601503

$-3.11001540379339$
0.18356682022241

$-1.56432609980280$

2.37009700934504

3.34846205638576

2.11431556876221

2.46718505671761

$-1.73774157210903$

$-2.48982380570524$

$-1.77084043590540$

$-2.02979000460737$

1.04343763575797

1.13375508246655

$-0.03743963211707$

1.48644897880544

2.06233318287156
0.18174824998397

$-0.31552931873570$

1.25072629030841

0.13389196131929

1.40737509057735

$-0.69160455801905$

1.87385754695988

2.03951746130729

$-0.24132447320609$

$-1.68135830811829$

1.05295174843499

2.86493310766919

$-0.89535393153131$

1.86854516249107

2.24751697394295

1.07439168841776

2.35146060181848

2.97308457343543

2.97438805528651

0.26502089130172

0.81192470053920

$-0.19228703269705$

$-0.83638641819670$

$-1.29484486816946$

$-0.47531160144444$

$-1.60930131749626$

2.98520110862170

3.19824627337316

2.67050413796392

3.91290073032778

1.95165044249730 

H 4.73668908724833
H 3.86913486268242
H 2.98817695897009
C 3.26025520712307
C 2.25416082019443
C 4.41241347023486
H 1.38208005195627
H 5.19757064091814
C 2.37805540530381
C 4.54604473816661
H 5.43496284722257
C 3.54290250661592
H 3.65384046369837
H 3.15136903825677
C 1.33961926584990
H 1.56948576865818
C -0.04162700267618
$\mathrm{H}-0.34591983896848$
H -0.74370666567158
O $\quad-4.69740719659909$
C -5.61048860259148
H -5.89122219715701
H -6.49409384316251
H $\quad-5.18594403909888$
$\mathrm{G}=-861719.0072 \mathrm{kcal} / \mathrm{mol}$
$\mathrm{N}_{\text {imag }}=1$
$-3.23158218248810$
$-3.71275087199261$
$-3.51112430145296$
$-1.81842079786804$
$-1.96363299458252$
$-2.60733007935230$
$-1.31753886926015$
$-2.48858112874663$
$-2.90154836778780$
$-3.54797098506850$
$-4.17133173689530$
$-3.69094605432280$
$-4.42664855589764$
$-1.07485782261157$
$-3.08328605550452$
$-3.81032380523961$
$-2.86854178006103$
$-2.54378911568878$
$-3.53460505646545$
$-0.33796756341491$
0.43003278474523
$-0.10044683292294$
0.56730273001757
1.40896410424939

\section{TS-p-OCF 3}

Pd 1.19464170125467

I 0.70444114161651

N 3.37960423450192

C -0.87439955978067

C -1.27909043790037

C -1.78325874021612

C -2.52641215840624

H -0.61640946326852

C -3.04557417250846

H -1.49513923272582

C -3.40090097604139

H -2.84052209194539

H -3.71496684409442

C 3.39136032050795

H 4.41493505683020

H 3.17134682496976

C 3.59625518406857

H 2.71087986735080

H 4.44715880552490

C 4.47750226656833

H 5.44260074869940

H 4.40575656967905
$-1.00758102590555$

1.65917918706016

$-0.67404569484926$

$-1.38204079693431$

$-1.80312514813992$

$-0.67569712282678$

$-1.44539220893596$

$-2.41063758710324$

$-0.33262209414668$

$-0.35000386873298$

$-0.71051938250529$

$-1.74356727826038$

0.24081555162148

0.68394394581406

0.87592760867647

1.40128861632710

$-1.66684016527994$

$-1.63585740565589$

$-1.31257356351371$

$-0.77025343352159$

$-0.72805676442747$

$-1.75377478704681$

1.40265489433207

2.86388602275165

1.34639281510678

$-3.68410449129113$

$-2.73633131228317$

$-3.62286592720750$

$-2.77999415170030$

$-4.36453928051520$

$-1.69017964952858$

$-2.60027113077078$

$-2.54483856469590$

$-1.64496930561412$

$-0.85274068571916$

$-4.46919984837438$

$-0.67159236489806$

0.10323498101530

$-0.92306686251772$

$-1.91232169345326$

$-0.43805821220291$

1.59647296055512

0.81412664237725

$-0.10576316454097$

1.44008660575113

0.55605527646086

0.18779009037421

$-0.26915045959767$

1.24687160477909

0.14653271721057

1.42592960492326

$-0.65143989562906$

1.92565470782878

2.03730961882107

$-0.17153177832848$

$-1.64501356495796$

1.12342648781261

2.92115264680127

$-0.79980371115435$

1.87079787943513

2.23809206531498

1.08195849257199

2.33972372703669

2.97753387205502

2.94840352698435

0.24743636968975

0.78455320312561

$-0.21603800501820$ 


$\begin{array}{cccc}\mathrm{C} & 4.44483202443200 & 0.28904131199484 & -0.84550171665458 \\ \mathrm{H} & 3.45098627599052 & 0.35950814684608 & -1.29378867594467 \\ \mathrm{H} & 4.72237693914888 & 1.28074787041795 & -0.47963030888120 \\ \mathrm{H} & 5.15516881442652 & 0.00385880107567 & -1.62775765209710 \\ \mathrm{C} & 2.38834846756900 & 0.88014288684854 & 3.00017574957862 \\ \mathrm{H} & 2.31425523313717 & 1.95019179836883 & 3.21730717941848 \\ \mathrm{H} & 1.39218753326170 & 0.54368774444306 & 2.69640022844796 \\ \mathrm{H} & 2.67744561088977 & 0.36842797333166 & 3.92295259858443 \\ \mathrm{C} & 3.85946538082983 & -3.10592704447045 & 1.92862215092467 \\ \mathrm{H} & 4.78324702655262 & -3.22250756190702 & 1.35700393127707 \\ \mathrm{H} & 3.95262758535794 & -3.71156093570993 & 2.83669068604782 \\ \mathrm{H} & 3.03426584143643 & -3.50761097669736 & 1.34188731799566 \\ \mathrm{C} & 3.28464851549886 & -1.78179984761389 & -3.70644362445698 \\ \mathrm{C} & 2.28450686218427 & -1.94660162050252 & -2.75578267475033 \\ \mathrm{C} & 4.44730268610278 & -2.55590079667338 & -3.65453925647618 \\ \mathrm{H} & 1.40265421219602 & -1.31353695848812 & -2.79370370704329 \\ \mathrm{H} & 5.22710018748333 & -2.42260317027328 & -4.39930492006610 \\ \mathrm{C} & 2.42638904685828 & -2.88898251513981 & -1.71572820688020 \\ \mathrm{C} & 4.59862756025103 & -3.50099654246622 & -2.63836266154434 \\ \mathrm{H} & 5.49601218670075 & -4.11262140648229 & -2.59118649415413 \\ \mathrm{C} & 3.60225336007671 & -3.66287053491305 & -1.67945272389333 \\ \mathrm{H} & 3.72628664468469 & -4.40113360951076 & -0.89160080028744 \\ \mathrm{H} & 3.16227662750289 & -1.03596120773556 & -4.48724833811195 \\ \mathrm{C} & 1.39801585466047 & -3.08988909083663 & -0.69247649399755 \\ \mathrm{H} & 1.63781202910231 & -3.82822327960349 & 0.06846273069968 \\ \mathrm{C} & 0.00964390203237 & -2.87755110353862 & -0.92908932544979 \\ \mathrm{H} & -0.29966976973525 & -2.54624634442943 & -1.91482688499484 \\ \mathrm{H} & -0.67637452417650 & -3.57432607855523 & -0.46372065736914 \\ \mathrm{O} & -4.62659231633919 & -0.42301288829477 & 1.73767815691793 \\ \mathrm{C} & -5.62685288631148 & 0.22111570658270 & 1.08683503961620 \\ \mathrm{~F} & -6.04834943146006 & -0.44075974592707 & -0.01970231869233 \\ \mathrm{~F} & -6.65823229176289 & 0.30992072346148 & 1.93784757655444 \\ \mathrm{~F} & -5.28844615726649 & 1.47411623368464 & 0.70093398060386 \\ \mathrm{G}=-1048603.552 \mathrm{kcal} / \mathrm{mol} & & \\ & & & \\ \mathrm{N} \text { imag }=1 & & & \\ & & & \end{array}$

